comme nous l'avons dit, à la première équation. (a) d'équilibre :

$$
h \theta+a t=\tilde{\omega} \rho
$$

où nous donnons à $\theta$ la valeur constante de $23 \mathrm{~kg}$. et à $h \theta$ la valeur de $8 \times 23$; cette équation s'écrit :

avec

$$
\begin{gathered}
8 \times 23+11,8 t=600 \tilde{\omega} \\
\tilde{\omega}>1 \mathrm{~kg} .32 .
\end{gathered}
$$

Nous pouvons ainsi établir le tableau suivant qui nous permet

\begin{tabular}{|c|c|c|c|}
\hline$\ddot{\omega}$ & $\theta$ & $t$ & \\
\hline 0,00 & $-12,4$ & 8,25 & \\
\hline 0,10 & $-9,6$ & 11,52 & \\
\hline 0,20 & $-7,0$ & 14,80 & \\
\hline 0,30 & $-4,4$ & 18 , & \\
\hline 0,45 & 0,00 & 23 , & \\
\hline 1,00 & 14,5 & 41, & \\
\hline$-1,2$ & 20 & 47,5 & $\begin{array}{l}\text { régime de } \\
\text { marche }\end{array}$ \\
\hline$-1,32$ & 23 & 51,40 & $\begin{array}{c}\text { limite d'élas- } \\
\text { ticité de la } \\
\text { tôle }\end{array}$ \\
\hline 1,50 & 23 & 60,6 & \\
\hline 2 & 23 & 86 , & \\
\hline$-2,40$ & 23 & 106,6 & $\begin{array}{r}(100 \% \text { de } \\
\text { surpression) }\end{array}$ \\
\hline 3 & 23 & 136 & \\
\hline$-3,45$ & 23 & 160 & rupture. \\
\hline
\end{tabular}
de suivre les relations entre les pressions et tensions de l'état de repos à la rupture.

Le graphique (2) est la représentation de ces relations.
A la surpression de 100: \%, la tension des fils $106 \mathrm{~kg}$. est encore loin de sa limite d'élasticité 140 à 150 . Le coefficient de sécurité est supérieur à 3 .

Le croquis (1) donne les états du tuyau.

$$
\begin{aligned}
& \text { Au repos .................. } \ddot{\omega}=0 \\
& \text { En régime de marche............ } \quad \tilde{\omega}=1 \mathrm{~kg} .20 \\
& \text { Sur } 100 \% \text { de surpression......... } \quad \vec{\omega}=2.400 \text {. }
\end{aligned}
$$

Le frettage comprendra 5 rangs de fil de $3 \mathrm{~mm}$. d'une épais. seur de $11 \mathrm{~mm}$. 8 .

L'épaisseur totale est donc de $8+11,8=20 \mathrm{~mm}$.

Les croquis (3) et (4) donnent la pression des fils en régime de marche $(\ddot{\omega}=1 \mathrm{~kg} .2)$ et au repos $(\dot{\omega}=0)$.

Le croquis (5) est la courbe des tensions de pose de chaque rang de fil, par lesquelles on obtient l'égalité des tensions sous pressions, constituant le "frettagc rectangulaire ".

Le poids de $1 \mathrm{~m}$. de ce tuyau est:

Tôle $237 \mathrm{~kg}$. $358 \mathrm{~kg}$, total $=595 \mathrm{~kg}$.

qui doit représenter 30 à $40 \%$ du poids d'un pareil tuyau établi en tôle bandagée.

La théorie du tuyau fretté à fil d'acier repose sur une analyse exacte de l'élasticité. Rien n'y est laisssé à l'approximation ou à l'empirisme.

On suit le développement successif des actions respectives. entre la tôle et le frettage sous les pressions croissantes jusquä éclatement.

Cet ensemble de propriétés réside principalement dans l'emploi d'un métal à la plus haute résistance et au dispositif en domnant l'utilisation au maximum de rendement.

\title{
Installation d'accumulation hydraulique du Lac Noir
}

\author{
par E. JAQUeT.
}

L'accumulation hydraulique par pompage est actuellement l'objet de plusieurs projets en France; l'installation des lacs Blanc et Noir dans les Vosges en est la première grande réalisation. La Société des Forces Motrices du Haut-Rhin avait déjà reconnu depuis de nombreuses années l'intérêt de combiner une usine travaillant au fil de l'eau sur le Rhin avec une installátion d'accumulation par pompage; l'usine du lac Noir a été construite dans ce but, pour valoriser les résidus d'énergie de l'usine de Kembs construite par la Société Energie Electrique du Rhin, usine dont l'inauguration a eu lieu l'automne dernier, en présence du Président de là République.

A l'ouest de Colmar, presque sur la crête des Vosges, se trouvent deux bassins naturels, les lacs Blanc et Noir, ayant une différence de niveau d'environ $115 \mathrm{~m}$. en moyenne et situés à une distance horizontale d'environ $1 \mathrm{~km}$. l'un de l'autre. Le massif séparant ces deux lacs est entièrement en granit. Ces conditions locales favorables, ainsi que la proximité de réseaux de distribution importants, conduisirent à choisir cet endroit pour l'établissement d'une installation d'accumulation hydraulique par pompage. Au début des travaux, une nouvelle société a été constituée, la Société Hydro-Electrique des Vosges, avec siège social à Mulhouse, pour s'occuper de la construction et de l'exploitation de cette installation; cette Société a confié l'exécution des travaux de génie civil à un Consortium de trois grandes entreprises (Société des Grands Travaux de Marseille,
Société Maison Fougerolles Frères, Société Générale d'Entre. prises).

$$
\text { I. - GÉNÉRAlités. }
$$

Les figures 1 et 2 (1) représentent le plan et le profil de l'ins. tallation. L'eau venant du réservoir amont, le lac Blanc, passe par une galerie bétonnée de $5 \mathrm{~m} .50$ de diamètre et une conduite forcée de $4 \mathrm{~m} .60$, disposée en partie en galerie dans le rocher, puis, après avoir traversé les turbines, elle est restituée dans le bassin aval, le lac Noir. L'eau refoulée par les pompes parcourt le même circuit en sens inverse.

Les deux lacs, de formation glaciaire, avaient été aménagés antérieurement au moyen de barrages pour servir de réservoirs régulateurs aux ruisseaux Blanc et Noir. Après l'exécution de l'installation des lacs Blanc et Noir, les réserves annuelles constituées jusqu'à présent dans chacun d'eux, en vue de la régt larisation de ces deux ruisseaux seront assemblés toutes deux dans Ie lac supérieur (lac Blanc).

En période d'exploitation, le niveau du lac Blanc subira d'une part des variations saisonnières qui suivront l'utilisatiol et la reconstitution périodique des réserves destinées à l'aljmentation des ruisseaux Blanc et Noir, d'autre part, des variations journalières dues au va-et-vient entre les deux lacs des tranches d'eau utilisées pour l'exploitation de l'usine du lac Noir.

(1) Voir page suivante. 
La différence de niveau maximum, qui pourra se produire au cours d'un cycle annucl dans le lac Blanc, par suite de ces deux causes de variation, pourra alleindre exceptionnellement $18 \mathrm{~m} .60$, mais restera sensiblement inférieur à ce chiffre pour une année normale.

Le niveau du lac Noir, par contre, ne subira que des variations journatières dues au déplacement entre les deux lacs des volumes d'eau ulilisés pour l'exploitation de l'usine du lac Noir. Le jeu de ces variations dépendra du régime d'exploitation de l'usine
Les deux lacs alimentaient jusqu'à présent deux vallées différentes. Pendant l'exploitation, l'écoulement direct du lac Blanc dans le ruisseau Blanc sera supprımé et l'alimentation des ruisseaux Blanc et Nolr se fera par l'intermédiaire d'une station de réglage placée en aval du lac Noir; cette dernière permettra de fournir aux deux ruisseaux les mêmes débits qu'avant la réalisation de l'mstallation lac Blanc-lac Noir.

De la station de réglage, l'eau destinée au ruisseau Blanc s'écoulera par une conduite en ciment débouchant dans ce

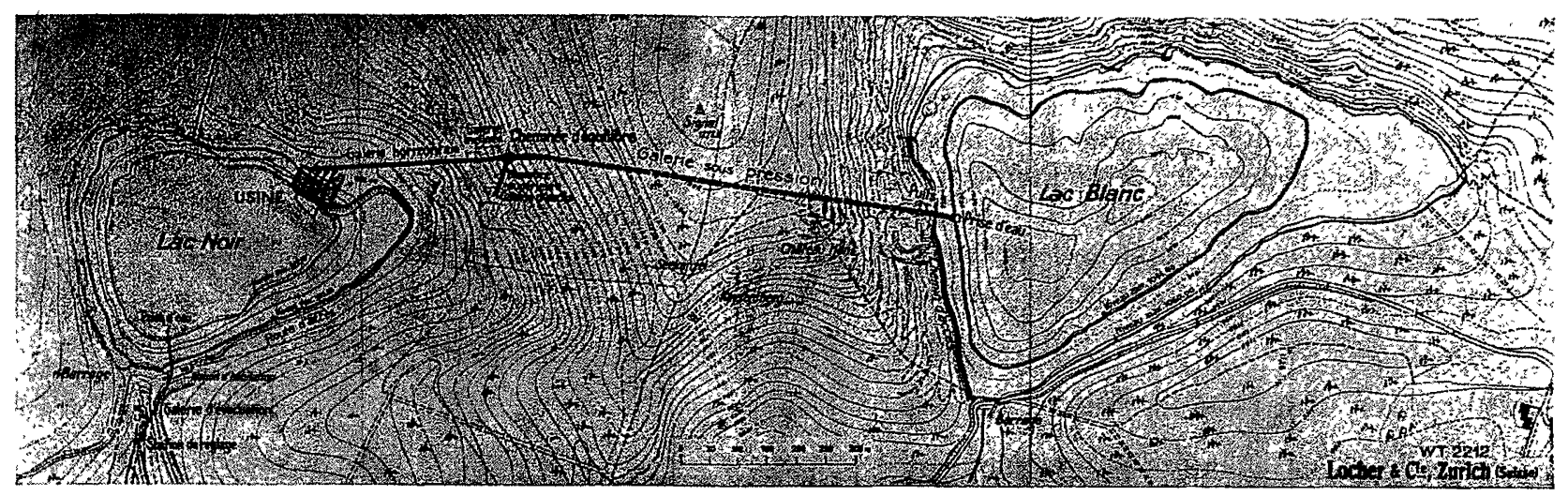

FIG. 1. - Plan de l'installation d'accumulation des lacs Blanc et_Noir.

et ne sera limité que par la cápacité utile du lac Noir correspondant à une tranche d'eau de $18 \mathrm{~m} .50$ de hauteur.

Il en résultc que la différence de niveau entre les dcux lacs subira des varialions saisonnières et des variations journalières, la différence de niveau pouvant alteindre les valeurs extrêmcs survantes :

$128 \mathrm{~m}$. lorsque les réserves saisonnières des deux ruisseaux seront entièrement constituées et que la tranche d'eau réservée à l'accumulation par pompage sera entièrement remontée dans le lac Blanc;

$90 \mathrm{~m}$. 80, lorsque les réserves saisonnières des ruisseaux seront entièrement épuisées (ce qui ne se produira qu'à la suite d'années très sèches) et que la tranche d'eau réservée à l'accumulation par pompage sera entièrement descendue dans le lac Noir.

Le volume d'eau maximum que l'on pourra utiliser pour l'exploitation de l'usine et qui correspondra à la capacité utile du dernier à environ $600 \mathrm{~m}$. ell aval du lac Blanc.

Sauf en ce qui concerne la section du ruisseau Blanc, situéc entre le lac Blanc et le débouché de la-conduite en question, les conditions d'écoulement de ce ruisseau ne seront ainsi pas changées.

Pendant la période de construction, l'alimentation. des deux vallées s'est faite de la façon suivante : l'exécution des travaux de l'usine nécessitait l'abaisscment par pompage dị niveau du lac Noir, jusqu'à plus de $25 \mathrm{~m}$. au-dessous de l'ancien exutoire du lac, de sorte que le ruisseau Noir serait resté sans eau. L'apport principal du lac Noir a été capté et dérivé par une rigole en tôle directement dans le ruisseau Noir. Mais ce débit étant très souvent insuffisant, notamment en èté, et le lac Noir ne pouvant plus, par suite de son abaissement, servir de bassin de compensation, on a été amené à prévoir d'utiliser la réserve d'eau du lac Blanc pour fournir les apports de débit nécessaires. Dans ce but, on a construit une rigole en tôle reliant le lac Blanc au

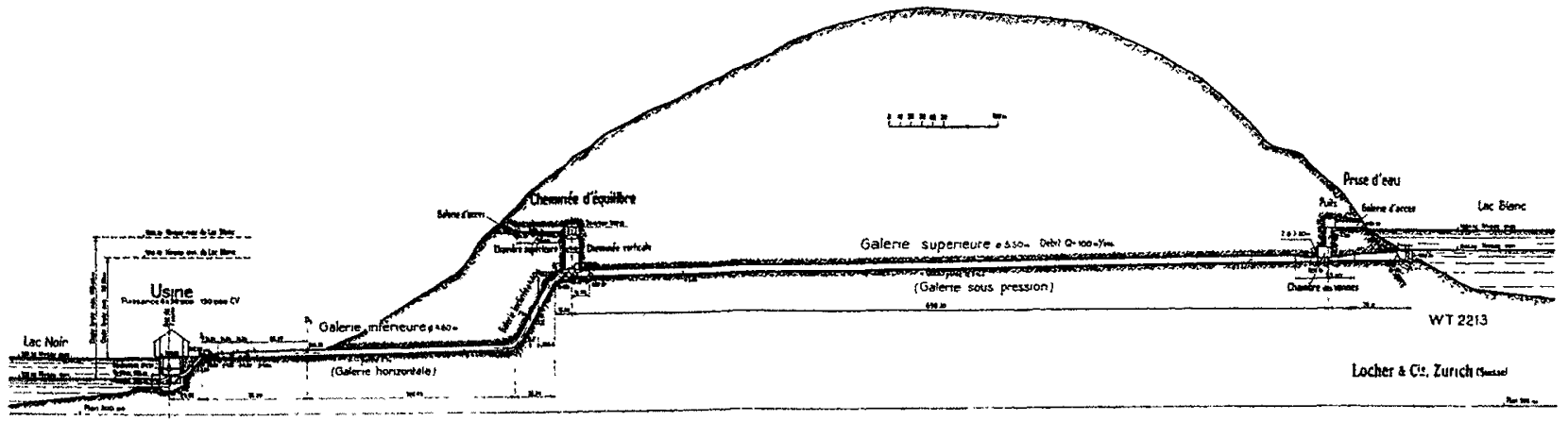

FIg. 2. - Profil en long de l'installation d'accumulation des lacs Blanc et Noir.

lac Noir, sera de $2.000 .000 \mathrm{~m}^{3}$ environ. Il permetira la marche à pleine ouverture des lurbines pendant 6 à 7 heures; pour le refouler du lac inférieur dans le lac supérieur, il faudra environ 12 à 13 heures de pompage à pleine charge.

(1) La maison Locher et Cie, à Zurich, qui a été choisie comme ingénieur-conseil par la Société Hydro-Electrique des Vosges et qui a 'établi d'autre part les plans de génie civil pour le compte du Consortium d'entreprises chargé de ces travaux, nous a cédé gracieusement les plans pour cet article, avec l'accord de la Société Hydro-Electrique des Vosges. ruisseau noir. Celte installation a cncore eu un autre but : Au commencement de l'hiver 1932, lorsque les travaux de l'usine furenit suffisamment avancés, el que les batardeaux servant à obturer les ouvertures des tuyaux d'aspiration des pompes ct des turbines furent mis en place, on a procédé au remplissage du lac Noir, en y emmagasinant, à l'aide de la rigole visée ci-dessus, la tranche d'eau du lac Blanc qu'll a fallu évacuer pour l'exécution des travaux de la prise d'eau.

Le niveau du lac Blanc a été abaissé jusqqu'à une cote un peu 
inférieure à la cote minimum d'exploitation. de telle sorte que les ouvrages de la prise d'eau puissent ètre construits cntièrement à sec.

L'exécution de la prise d'eau par une autre mélhode, comme par exemple sous caisson à l'air comprimé, se serail heurtée à de grosses difficultés dues aux conditions géologiques et topographiques locales défavorables, et, partant, aurait conduil à un prix prohibitif.

Le programme d'exéculion des liavaux du lac Blanc a été élabli de manière que lors de la mise en service de l'usine, on dispose, dans le lac Blanc, d'un volume d'eau suffisant pour permellre de réaliser les différents essais et mises au point nécessaires pour que l'on puisse procéder au pompage de la tranche d'eau accumulée dans le lac Noir. Les pompes ne pouvant, en effet, pas démarrer directement avec les alternateurs travaillant comme moteurs synchrones, il esl nécessaire de lancerd'abord le groupe à l'aide de la turbine. Lorsque le synchronisme est atteint, la mise en parallele peut se faire cl le pompage commencer.

\section{2. - Chanbre des vannes et condutte forcié.}

L’équipement complel de la chambre des vannes, ainsi que toutc la conduite forcée, sont fournis par la Soziélé Alsacienle de Constructions Mécaniques à Mulhouse, avec le concours le:hnique de la $\mathrm{S}$. A. des Ateliers de Constru-lions Mécaniques Escher Wyss, à Zurich.

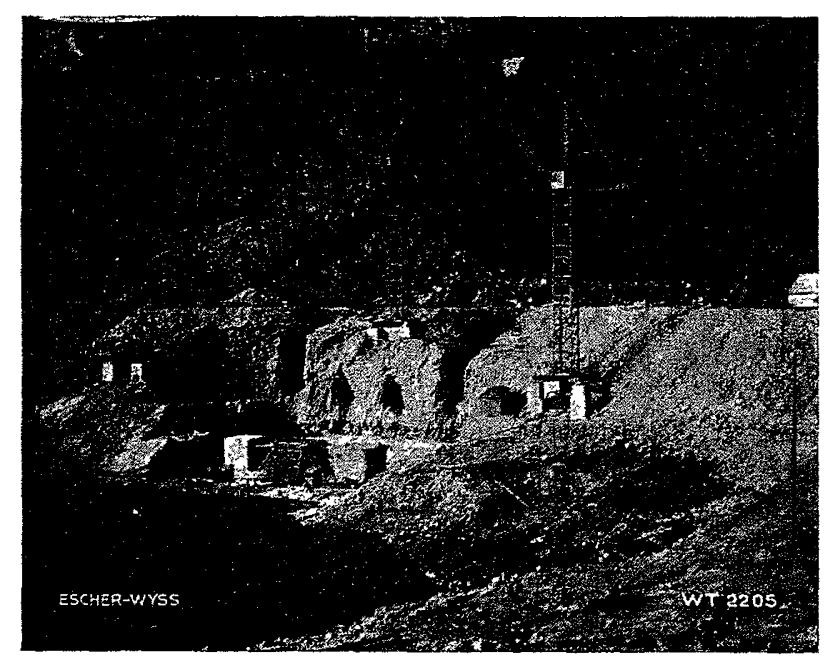

Frg. 3.- Excavation de l'usine au lac Noir (niveau du lac abaissé à la cote 923 ).

Le travail est réparti entre ces deux maisons de la façon suivante : Escher Wyss a fait tous les calculs et dessins de construction et a pris à sa charge tous les travaux de soudure sur le chantier, ainsi que le montage et quelques fournitures accessoires. La Société Alsacienne fournit les demi-viroles cintrées et chanfreinées, toutes les vannes à papillon et la grille placée dans la conduite forcée.

La chambre des vannes comprend deux tronçons de conduite de $3 \mathrm{~m}$. 20 de diamètre en tôle soudée reliant les deux galeries de la prise d'eau à la galerie sous pression de $5 \mathrm{~m}$. 50 de diamètre ; chacun de ces trọnçons est muni de deux vannes à papillon avec commande par moteur électrique et à la main. Les vannes d'aval sont, en outre, munies d'un mécanisme de fermeture rapide par contrepoids déclenché automatiquement depuis l'usine par un électro-aimant en cas d'accident.

On a prévu deux tronçons de conduite d'une part pour réduire les dimensions des vannes et ensuite pour obtenir de bonnes conditions hydrauliques pour les essais de réception des tur- bines el des pompes. En effet, on a prévu que pour ces essais, la mesure des débits se ferait au moyen de moulinets placés dans l'un des tronçons de conduite entre les deux vamnes. Pendant l'essai, les vannes de l'autre conduite seront fermées, de sorte que la vitesse de l'eau, même à charge réduite pour une seule

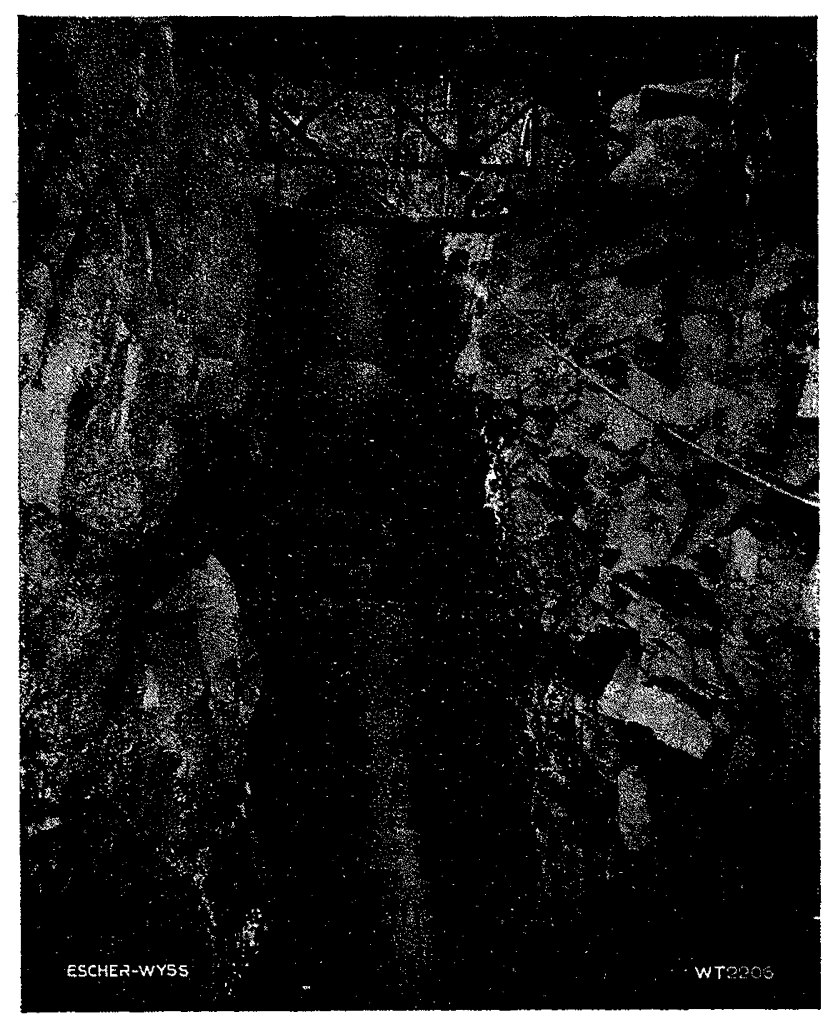

Firs. 1. - Montage d'unz concluite d'alimentation.

pompe, sca cuncore suefisamment grande four pe miclle d'obleni i des résuitals sûrs avee les moulinets.

Certans accessoires de la chambre des vannes, leis que les deux groupes moto-jompes d'épuisement de 12 litres/sec. chacun, les deux ponts roulants de 10 tonnes, placés l'un en haul du puils d'accès dans la chambre des vannes el l'autre dans la chambre des vannes mêmes, le manomètre destiné à transmettre à l'usine du lac Noir les niveaux du lac Blanc, ansi que lout l'équipement élect.ique des vannes, des molo-pompes el des ponts-roulants sont fournis par la Sociélé Alsacienne de Constructions Mécaniques et la Société Escher Wyss.

Les deux conduiles se réunissent à l'aval de la chambre des

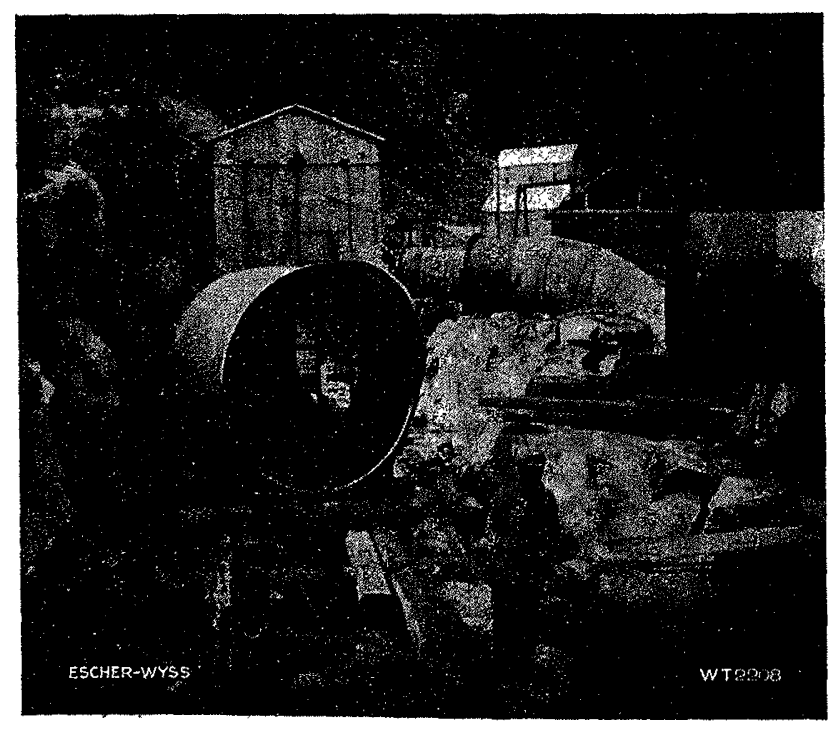

Firi. j. Montage lles courles supérieurs. 
vamnes et se raccordent à la galerie bétonnée de $5 \mathrm{~m}$. 50 de diamètre, qui conduit en ligne droite et avec une pente constante de $12,5 \%$ à la cheminée d'équilibre. Celle-ci est de construction spéciale, avec étranglement à la base du puits et déversoir à la partie supérieure. Avec cette disposition, on obtient un puits de section plus faible qu'avec la construction normale. Toutefois, une ouverture de $13 \mathrm{~m}$. de diamètre est encore nécessaire. Les maisons Locher et Cie et Escher Wyss, à Zurich, onl fait des études approfondies sur l'influence mutuelle des variations de niveau dans la cheminée sur le réglage des turbines et pompes, et les coups de bélier. Les conditions d'exploitation de l'usine du lac Noir ont permis de renoncer à prévoir une chambre inférieure à la cheminée d'équilibre, sans diminuer, pour cela, la sécurité de l'installation.

Le premier projet prévoyait l'installation de quatre conduites forcées à ciel ouvert. Ce projet fut ensuite modifié pour diverses de la cheminée d'équilibre, à laquelle elle se raccorde par un coude, puis d'une partie, rectiligne également, à faible inclinaison, de $2 \%$ de pente, reliée à la première par un coude (voir fig. 2). Ce tracé a été choisi pour que la plus grande partie de la conduite soit placée à une profondeur suffisante dans du bon granit. Ce tracé qui, au point de vue des travaux de génie civil et du montage de la conduite, est d'une exécution plus difficile qu'un tracé avec puits vertical, présente l'avantage sur ce dernier d'être moins long et de diminuer les pertes hydrauliques aux coudes.

La conduite entière est construite en tôle d'acier et a étéapprovisionnée en éléments de demi-viroles de $2 \mathrm{~m}$. 75 de longueur Les viroles de $5 \mathrm{~m}$. 50 de longueur, dont se compose la conduite ont été fabriquées dans un atelier sur le chantier, en assemblant quatre de ces éléments par soudure électrique. Le blindage commence quelques mètres en amont de la cheminée d'équi-

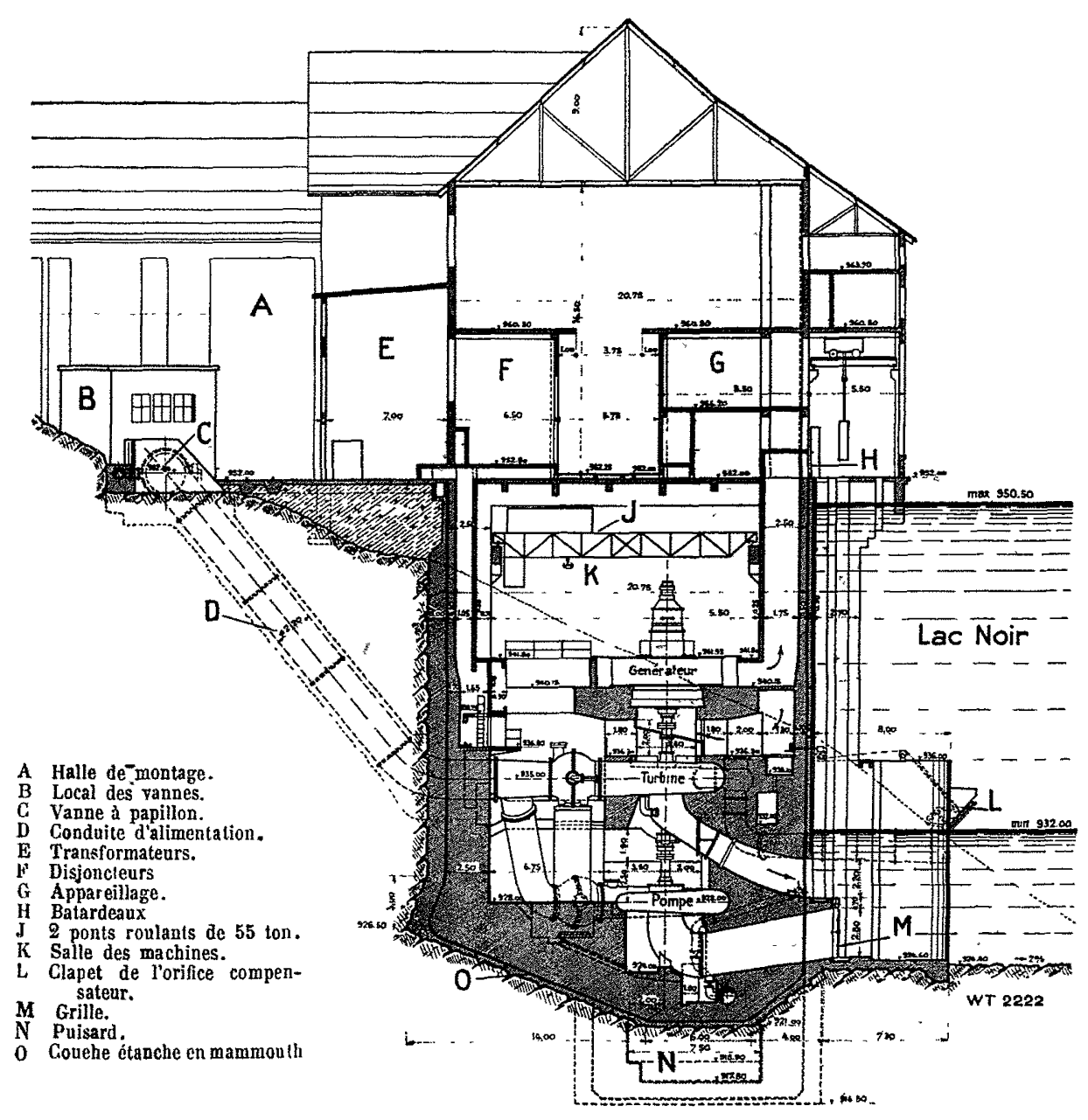

Fig. 6. - Coupe de l'usine.

raisons, entre autres pour respecter davantage la beauté du site du lac Noir et l'on décida de remplacer jusqu'au collecteur les quatre conduites par une conduite unique, placée en partie dans le rocher. Une solution semblable a déjà été adoptée dans d'autres installations, par exemple à la Handeck (Suisse), Galleto (Italie) Achensee (Tyrol), La Truyère (France), installations qui ont toutes été faites par Escher Wyss.

Le diamètre de cette conduite est de $4 \mathrm{~m}$. 60, ce qui donne, pour un débil de $100 \mathrm{~m}^{3} / \mathrm{sec}$., une vitesse de $6 \mathrm{~m}$./sec. Avec la conduile unique, on a le grand avantage par rapport à la solution prévoyant qualre conduites de faible diamètre, d'avoir des pertes de charge plus faibles, surtout lorsque l'installation ne marche pas à plein débit.

Le profil de la conduite forcée est composé d'une partie rectiligne inclinée à $60^{\circ}$, commençant immédiatement en aval libre. Entre la tôle de la conduite forcée et le rocher, il y a un espace d'environ $30 \mathrm{~cm}$., sauf dans les coudes et au droil du raccordement avec la cheminée d'équilibre où l'espace en quesLion est plus grand. La conduite a été enrobée de béton, virole par virole, après l'achèvement de chacune des soudures transversales, pour avoir la garantie d'obtenir un béton compact. sans vides.

En outre, on a exécuté, après le montage, des injections de ciment à travers la tôle, entre la tôle et le béton d'une part, et entre le béton et le rocher d'autre part. Cette précaution est importante parce que, sans l'appui du béton et du rocher, la tôle serait chargée jusqu'à sa limite d'élasticité. A vrai dire, la conduite métallique dans la première partie n'est qu'un blindage et non une conduite forcée proprement dite, puisqu'elle ne doit supporter qu'une partie de la pression de l'eau, l'autre 
partie étant transmise au rocher par l'intermédiaire de l'enrobage de béton. On a estimé que le rocher supporlerail au moins la moitié des efforts, tant que l'épaisseur de la couche de rocher au-dessus de la conduite serail supérieure à $60 \mathrm{~m}$. Dès que le recouvrement de rocher devient inférieur à ce chiffre, les efforts susceptibles d'ètre supportés par la tôle augmentent au fur et à mesure qu'on arance jusqu'à une vinglaine de mètres en amont de la sortie du tunnel. En aval de ce point, la conduite est calculée pour supporter à elle seule toute la pression avec un coefficient de sécurité de quatre.

Le collecteur se compose égalemenl d'éléments en tôle soudés sur le chantier. Les trois pièces de dérivation représentant un travail de chaudronnerie extrêmement délicat, ont été assemblées provisoirement dans les ateliers d'Escher Wyss, à Zurich,

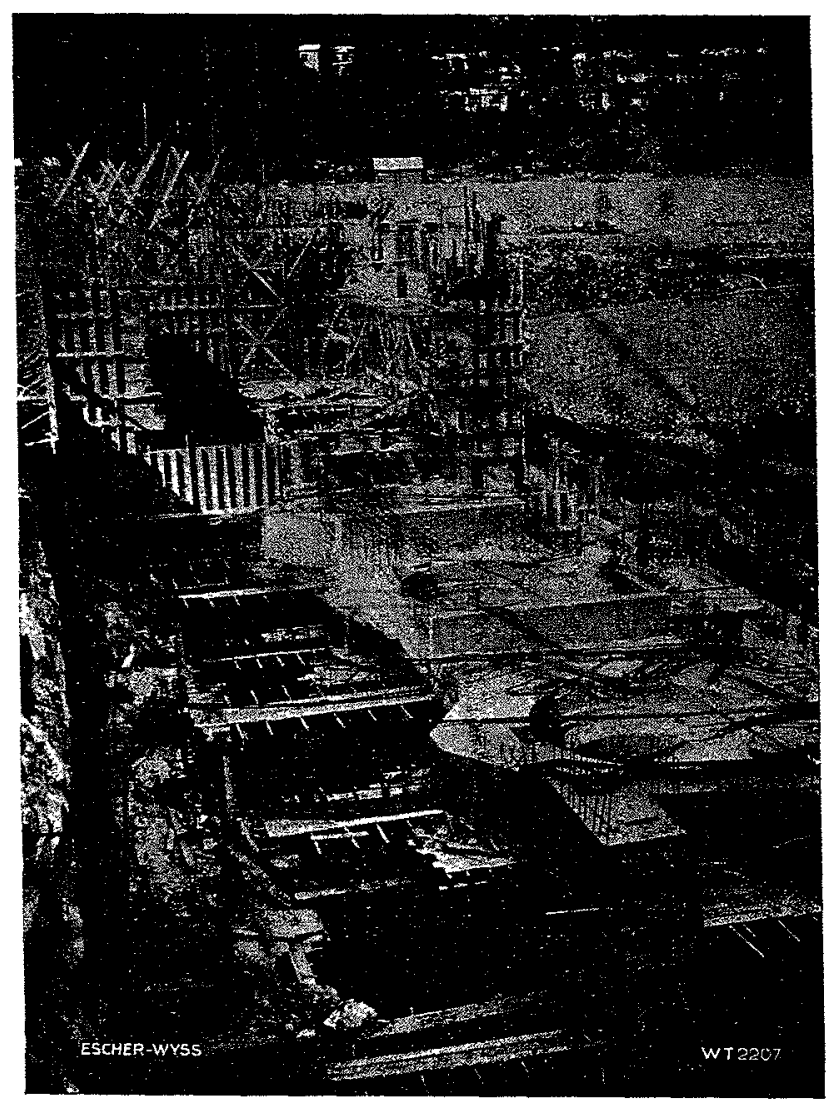

Fig. 7.- Usine en construction, plancher des alternateurs (cote 940,15$)$.

et soudées définitivement sur place. La conduite est munie d'un trou d'homme à son extrémité aval et d'une grille à son extrémité amont. Du collecteur partent quatre branchements de $2.200 \mathrm{~mm}$. de diamètre, reliant la conduite aux quatre groupes principaux de l'usine, ainsi qu'une petite conduite alimentant la turbine auxiliaire. En tête de chacune de ces quatre conduites, il y a une vanne à papillon prévue pour permettre la révision des vannes sphériques placées à l'entrée des bâches des turbines el des pompes. Les deux branchements extrêmes ont été posés en tranchée, ceux du milieu du Lunnel, ainsi qu'on le voit sur la figure 3. Les figures 4 el 5 représentent deux phases du montage des branchements. Le grand bâtiment visible sur la figure 5, à l'arrière-plan, est l'alelier de soudure. Au bas de chaque branchement se trouve une culotle en acier coulé pour son raccordement à la turbine et à la pompe.

$$
\text { 3. - USINE. }
$$

L'usine contient quatre groupes composés chacun d'une pompe, d'une turbine et d'une machine électrique, pouvant fonctionner comme généraleur el comme moteur. Par suite des grandes variations de niveau du lac Noir, il étail tout indiqué d'adopter une disposition à axe vertical. La figure 6 (1) représente une coupe de l'usine. Les caractéristiques principales des machines sont les suivantes :

4 alternateurs de $25000 \mathrm{kVA}$ chacun, tension $12000 \mathrm{~V}$; 4 turbines Francis de $24000 \mathrm{CV}$. chacune, sous la chute nette la plus faible de $85 \mathrm{~m}$. Pour des chutes plus élevées, la puissance peut augmenter selon la possibilité de charge des alternateurs. Sous la chule maximum, chaque turbine peut fournir une puissance à l'accouplement d'environ $40000 \mathrm{CV}$. Les allernateurs sont susceptibles de supporler une surcharge eonsidérable pendant un temps plus ou moins long, suivant la température de l'air ambiant, 'e qui est très importanl pour une usine de pointe.

3 pompes centrifuges d'un débil moyen de $13 \mathrm{~m}^{3} / \mathrm{sec}$, avec une hauteur de refoulement moyenne de $117 \mathrm{~m}$., absorbant une puissance de $27000 \mathrm{CV}$. chacune.

1 pompe centrifuge de $8 \mathrm{~m}^{3} / \mathrm{sec}$, sous la même hauteur de refoulement et une puissance de $17000 \mathrm{CV}$.

Tous les groupes tournent à la vilesse de 272,7 lours par minute.

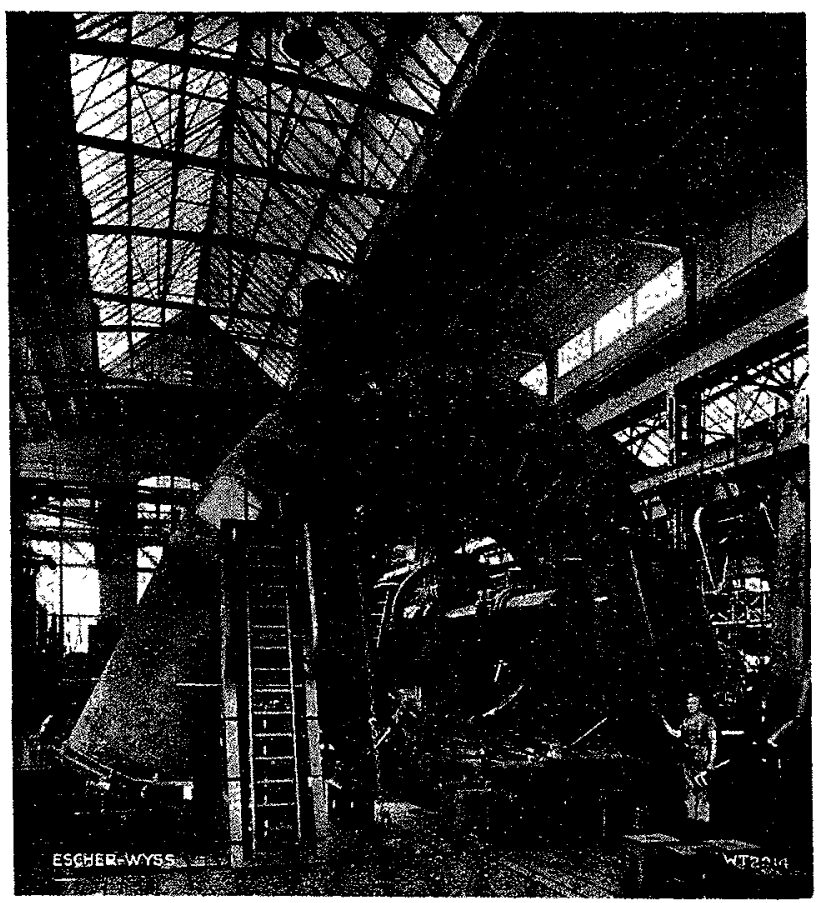

FIG. 8. - Bâche spirale de la turbine sur le tour.

La fourniture des turbines et des pompes des groupes d'acctmulation avec tous leurs accessoires, ainsi que la lurbine auxiliaire et les trois groupes moto-pompes d'épuisement, a été confiée à la maison Escher Wyss, à Zurich, avec l'obligation de faire exécuter en France une partie importante de celte fourniture; les allernateurs et les transformateurs sonl fournis par Alsthom.

Les points de vue suivants ont dû être respectés pour la disposition générale : Les cotes d'implantation des pompes ef tur* bines ont élé choisies de façon à ce que, pour le niveau minimum du lac Noir (932), le risque de cavitation soit tout à fait éliminé. Cetle condition exige une pression à l'entrée de la pompe de $4 \mathrm{~m}$. Des questions de construction onl défini alors la cote de

(1) Ce plan a été mis gracieusement à notre disposition par la maison Locher et Cie, à Zurich. 
la turbine, tout en respectant, pour elle aussi, les conditions de cavitation. La lurbine lravaille, par conséquent, avec aspiration si le niveau du lac Noir esl abaissé el avec contre-pression s'il se trouve entre les cotes 935 et 950,50 .

L'emplacement des allernateurs représente un fail nouveau dans la construction des usines hydraulicques. La règle suivant laquelle l'alternateur doit être placé au-dessus de la cote maximum du niveau aval a déjà été abandonnée pour quelques installations eonstruites au cours de ces dernières années. Mais, dans ces usines, le plancher de la salle des machines n'a été abaissé que de quelques mètres. Au lac Noir, par contre, on s'est décidé à installer des alternateurs $10 \mathrm{~m}$. en dessous du niveau maximum du bassin aval. Grâce à la réduction d'encombrement qui en résulte, une économie considérable a pu être réalisée. Lorsque le lac Noir est plein, la salle des machines, y compris les pontsroulants, est entièrement en dessous du niveau du lac; seule la partie du bâtiment qui abrite les transformateurs, les disjoncteurs et les sectionneurs reste toujours au-dessus de l'eau. J.es fondations du bâtiment et ses murs de pourtour sont protégés par une couche étanche d'un produit bitumineux nommé mammouth, fourni par la Société Alsacienne d'Emulsion, à Strasbourg. Les conduites et les tuyaux d'aspiration sont munis d'un dispositif spécial, à l'endroit où ils traversent la couche étanche, pour leur raccỡdement à cetle dernière, afin d'éviter loute infiltration. Pour protéger le mamouth, on l'a recouvert d'un enduit en ciment et d'une couche de béton. L'usine a été construite de façon à pouvoir, lorsqu'elle est entièrement immergée, supporter les poussées hydrostatiques considérables exercées sur ses murs de pourtour. Le poids de ses fondations ainsi qu'un ancrage de ces dernières dans le rocher, éliminent tout danger de soulèvement sous l'action des sous-pressions.

La figure 7 montre l'usine construite jusqu'au plancher des allernateurs.

Un autre délail intéressant réside dans l'accouplement de la pompe. Dans la plupart des usines actuellement en exploitation, la pompe est munie d'un accouplement débrayable, soit d'un type mécanique, soit d'un type hydraulique (1), comme c'est, par exemple, le cas à la centrale de Schluchsee. Au lac Noir, on a dû renoncer à ce dernier type d'accouplement, malgré les grands avantages qu'il présente, car il aurait impliqué un abaissement sensible du radier de l'usine. L'accouplement de ces groupes est rigide, de sorte que la roue de la pompe tourne également pendant la marche de la turbine. L'eau est expulsée de la bâche par de l'air comprimé et maintenue à un niveau constant dans le tuyau d'aspiration, grâce à un flotteur muni d'un réglage automatique. La pression de l'air à l'intérieur de la pompe varie suivant le niveau du lac Noir. Pendant le pompage, la turbine est vidangée de la même façon. Un dispositif de réfrigération par injection dans les interstices préserve les labyrinthes de l'échauffement créé par la marche dans l'air comprimé. Chaque groupe a son compresseur commandé par moteur électrique et deux réservoirs d'air à $25 \mathrm{~kg} / \mathrm{cm}^{2}$. Tous les réservoirs peuvent être mis en communication pour éviter toute interruption, si l'un des compresseurs devait ne pas fonctionner. Ils sont, en outre, largement dimensionnés de façon à suffire à plusieurs manœuvres consécutives. Ce système avec accouplement rigide a deux avantages : les groupes sont toujours prêts à passer d'un régime à l'autre et le pivot de la pompe peut être supprimé. Toutes les masses tournantes, le rotor de l'alternateur, les roues de la lurbine el de la pompe sont suspendus à un seul pivot,

(1) Voir l'article de G. Kuhne : "L'emploi de l'accouplement hydraulique pour la commande des pompes d'accumulation", dans le $n^{\circ} 5$ du "Bulletin Escher-Wyss", 1932, page 129. monté sur le croisillon supérieur de l'alternateur. C'ette disposition est préférable à celle du pivot monté entre la turbine et l'alternateur, parce que les dimensions du pivot en sont réduites, son accès est plus facile et, par conséquent, son contrôle plus régulier.

On pourrait craindre que le refoulement pneumatique ne présente pas la sécurité nécessaire, mais, avec le nombre de compresseurs et de réservoirs prévus, il n'y a aucun risque. La vidange des bâches dans le puisard aurait nécessité des organes d'obturation très délicats dans les tuyaux d'aspiration et un puisard avec de grosses pompes d'épuisement. Avec le système pneumatique, par contre, il suffit d'installer quelques petites pompes d'assèchement pour épuiser les eaux de pertes et d'infiltration.

Les roues des turbines et pompes sont en bronze, l'acier étant exposé à une forte oxydation, puisqu'elles tournent tantôt dans l'eau, tantôt dans l'air.

La figure 8 représente la bâche spirale de la turbine sur le tour. Elle est composée de segments en tôle soudée et sera transportée en quatre parties. Sur la figure 9 , on voit la pompe à l'état de montage dans les ateliers Escher Wyss.

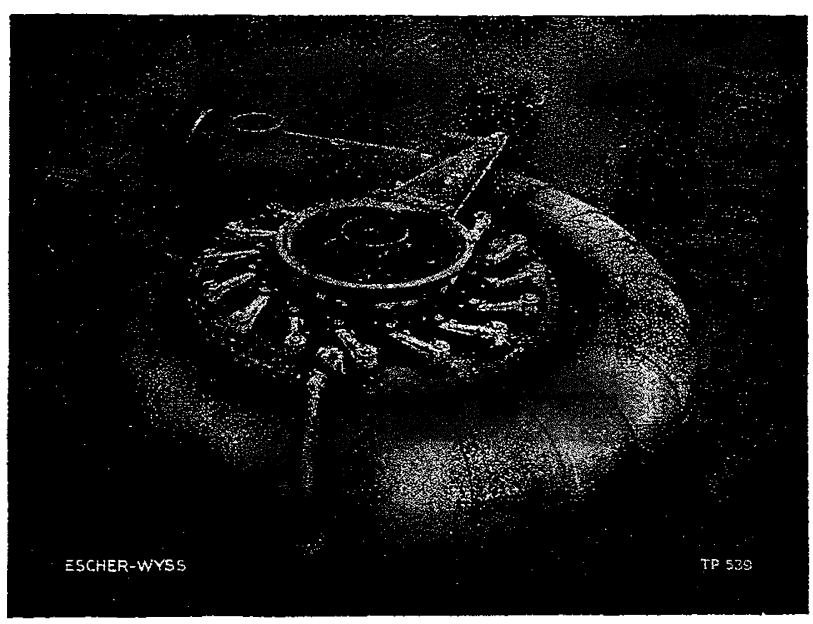

Fig. 9. - Pompe d'accumulation de $13 \mathrm{~m}^{3}$ /sec, au montage en atelier.

Les vannes rotatives montées à l'entrée de chaque turbine et à la sortie de chaque pompe, sont munies de servo-moteurs hydrauliques avec relais à huile sous pression. L'eau agissant dans les servo-moteurs est prise directement sur la conduite forcée. La commande se fait par électro-aimant, sur place ou à distance. Les ouvertures des tuyaux d'aspiration du côté du lac peuvent être fermées par des batardeaux, fournis par la maison Joya, à Grenoble. Pour la grille mobile, à l'entrée de la pompe, on emploie les mêmes glissières que pour les batardeaux. Lors d'une révision de la pompe, il faut donc retirer tout d'abord la grille avant de pouvoir descendre le batardeau. Les tuyaux d'échappement des orifices compensateurs sont munis de clapets. La commande des groupes est automatique, de sorte qu'on peut, par un seul mouvement au tableau, faire marcher les groupes à n'importe quel régime, soit pompage, soit marche en turbine avec création d'énergie électrique, soit encore marche de l'alternateur comme compensateur de phase, la turbine et la pompe tournant alors à vide. La charge des turbines et pompes sera réglée au début par boutons-poussoirs au tableau, mais toute la commande est prévue déjà maintenant pour s'adapter éventuellement plus tard, sans changement de construction, a une commande à distance.

La mise en service des deux premiers groupes de l'usine est prévue pour l'automne 1933. 